\title{
Preeclampsia: prevalencia y factores asociados en gestantes de una institución de salud de Boyacá en el periodo 2015 a 2017
}

\author{
Andrea L Salamanca-Sánchez ${ }^{1}$, Laura A Nieves-Díaz², Yury M Arenas- Cárdenas ${ }^{3}$ \\ RESUMEN
}

La preeclampsia es un trastorno hipertensivo en gestantes, tratado como una patología multisistémica que genera cambios vasculares y hemodinámicos.

Objetivo: Identificar la prevalencia y los factores asociados a preeclampsia en gestantes en una Institución de Salud, en los años 2015, 2016 y 2017.

Metodología: Estudio retrospectivo analítico de corte transversal en gestantes de una Institución de Salud, en los años 2015, 2016 y 2017. Se obtuvieron 2531 gestantes de la base de datos y se realizó un análisis de diferencias de medias por ANOVA y un análisis multivariado con regresión logística para calcular el OR con un IC al $95 \%$ y un $P<0,05$. En este estudio no se publica información que pueda vincular a las pacientes.

Resultados: La prevalencia de preeclampsia fue de 3,5\% y factores de riesgo asociados como la edad de 35 años o más, que tuvieron 5,2 veces más probabilidad de tener Preeclampsia en comparación con las menores de 35 años (OR: 5,2; IC 95 \%: 3,258-8,335). Las gestantes que tienen riesgo psicosocial alto tuvieron 2,1 veces más probabilidad de presentar preeclampsia en comparación con las que tienen un riesgo psicosocial bajo (OR: 2,134; IC $95 \%$ : 1,380-3,300).

Conclusión: Se encontró prevalencia de preeclampsia del 3,5 \% y se determinó que la edad es un factor de riesgo para sufrir preeclampsia, en especial mayores de 35 años.

Palabras clave: Embarazo, factores de riesgo, preeclampsia, prevalencia.

\footnotetext{
${ }^{1}$ Secretaría de Salud de Boyacá, Tunja, Colombia

${ }^{2}$ Alcaldía Municipio de Saboyá, Saboyá, Colombia

${ }^{3}$ Universidad de Boyacá, Tunja, Colombia
}

Correspondencia: Andrea L Salamanca-Sánchez Dirección: cra 6ª \#7ª-28 Velez Santander Celular: 3142755640

Correo electrónico: andrealsalamanca21@hotmail.com

Citar este artículo así:

Salamanca-Sánchez AL, Nieves-Díaz LA, Arenas- Cárdenas YM. Preeclampsia: prevalencia y factores asociados en gestantes de una institución de salud de Boyacá en el periodo 2015 a 2017. Revista Investig Salud Univ Boyacá. 2019;6(2): 40-52. doi: https://doi.org/10.24267/23897325.422 


\title{
Preeclampsia: prevalence and associated factors in pregnant women of a health institution of Boyacá in the period 2015 to 2017
}

\begin{abstract}
Preeclampsia is a hypertensive disorder in pregnant treated as a multisystem pathology that generates vascular and hemodynamic changes.

Objective: To identify the prevalence and factors associated with pre-eclampsia in pregnant women from an Institution of Health in 2015, 2016 and 2017.

Methodology: Cross-sectional retrospective analytical study of pregnant women from an Institution of Health in 2015, 2016 and 2017. Obtained 2531 pregnant women from the database and analyzed mean differences by ANOVA and a multivariate analysis with logistic regression to calculate the OR with $95 \% \mathrm{Cl}$ and $\mathrm{P}<0.05$. This study does not publish information that can link patients.
\end{abstract}

Results: The prevalence of pre-eclampsia was $3.5 \%$ and associated risk factors such as age 35 years or older, which were 5.2 times more likely to have pre-eclampsia compared to those under 35 years (OR: 5, 2; 95\% Cl: 3,258-8,335). Pregnant women with high psychosocial risk were 2.1 times more likely to have pre-eclampsia compared with those with a low psychosocial risk (OR: $2,134,95 \% \mathrm{Cl}$ : 1,380-3,300).

Conclusion: A prevalence of Preeclampsia of 3.5\% was found and it was determined that age is a risk factor for suffering Preeclampsia, especially those over 35 years old.

key word: Pregnancy, risk factors, pre-eclampsia, prevalence. 


\section{Pré-eclâmpsia: prevalência e fatores associados em gestantes de uma Instituição de saúde de Boyacá no período 2015 a 2017}

\section{RESUMO}

A pré-eclâmpsia é um transtorno hipertensivo em gestantes, tratado como patologia multissistêmica que gera mudanças vasculares e hemodinâmicas.

Objetivo: Identificar a prevalência e os fatores associados à pré-eclâmpsia em gestantes de uma Instituição de Saúde nos anos 2015, 2016 e 2017.

Metodología: Estudo retrospectivo analítico transversal em gestantes de uma Instituição de Saúde nos anos 2015, 2016 e 2017. Foram obtidas 2531 gestantes da base de dados e foram realizadas analises das diferenças de médias pela ANOVA e uma análise multivariada com regressão logística para estimar o OR com um IC de $95 \%$ e um $P<0,05$. Neste estudo não foi divulgada informação que possa vincular as pacientes.

Resultados: A prevalência da pré-eclâmpsia foi de 3,5 \% e fatores de risco associados como a idade de 35 anos ou mais, tiveram 5,2 vezes mais probabilidade de ter pré-eclâmpsia em comparação com as menores de 35 anos (OR: 5,2; IC 95 \%: 3,258-8,335). As gestantes que têm alto risco psicossocial tiveram 2,1 vezes mais probabilidade de apresentar pré-eclâmpsia em comparação com as que têm um baixo risco psicossocial (OR: 2,134; IC 95 \%: 1,380-3,300).

Conclusão: Encontrou-se prevalência de pré-eclâmpsia de 3,5 \% e determinou-se que a idade é um fator de risco para sofrer pré-eclâmpsia, em particular ás maiores de 35 anos.

Palavras-chave: Gravidez, fatores de risco, pré-eclâmpsia, prevalência. 


\section{INTRODUCCIÓN}

La preeclampsia (PE) es un trastorno hipertensivo en las gestantes, tratado como una patología multisistémica que genera cambios vasculares y hemodinámicos en la mujer (1). Para su diagnóstico se determinan criterios clínicos tales como: Edad gestacional, mayor a 20 semanas; tensión arterial (TA), mayor a 140/90 mmHg, acompañada de proteinuria ( $\geq 300 \mathrm{mg} / 24 \mathrm{~h}$ ), y sintomatología asociada como tinitus, cefalea, fosfenos, edemas en miembros superiores e inferiores en estado de reposo y epigastralgia (2).

Son considerados factores de riesgo para las mujeres en gestación: presentar edades extremas, antecedentes reproductivos (nuliparidad, multiparidad), obesidad, nivel socioeconómico bajo, ausencia de servicios profesionales de atención de la salud (3), desnutrición, antecedente familiares, enfermedades renales, entre otros (4).

De acuerdo con la declaración de los Objetivos de Desarrollo del Milenio (ODM), firmada por 189 estados miembros de las Naciones Unidas, varios países implementaron estrategias en salud con la finalidad de disminuir en tres cuartas partes la mortalidad materna asociada al embarazo, para el año 2015. Sin embargo, la mortalidad materna se ha convertido para algunos países en un reto no resuelto (5). De igual forma, los objetivos del desarrollo sostenible identifican la salud y el bienestar, así como la equidad de género, dentro de las estrategias que favorecen a la mujer durante la gestación, el parto, el puerperio y sus decisiones reproductivas (6).

En el contexto mundial, la incidencia de preeclampsia oscila entre 2-10\% de los embarazos; La Organización Mundial de la Salud (OMS) (7) estima que su incidencia es siete veces mayor en los países en desarrollo que en los desarrollados (8); en esta medida, la preeclamsia afecta alrededor de 1 de cada 2000 partos en países desarrollados, mientras en los países en desarrollo, esta cifra varía desde 1 en 100 a 1 en 1700 . Adicionalmente, según la OMS, la mortalidad materna es más frecuente en las zonas rurales y en las poblaciones más pobres; la anterior afirmación toma relevancia ya que el $50 \%$ de las mujeres gestantes en el mundo viven en zonas rurales, a más de $5 \mathrm{~km}$ de un hospital cercano y tienen, dificultades en el acceso a centros asistenciales de salud para la realización de controles prenatales o asistencia ginecobstétrica (9). En Colombia se implementó el Modelo Biopsicosocial para la reducción de la morbilidad y mortalidad materna y perinatal, el cual consiste en una metodología elaborada a partir de una investigación llevada a cabo entre 1987 y 1990, la cual fue validada en cuatro países de América Latina entre 1991-1992 (MBPS); dicho modelo implementó como un estándar nacional de la atención sanitaria, asimismo, se introdujo, como política de salud pública nacional (10). 
Agudelo y colaboradores evidencian en su estudio que el $90 \%$ de las gestantes que padecían preeclampsia presentaban signos y síntomas como cefalea severa, trastornos visuales y presencia de edema (11). Para "el año 2012 a 2013 del total de muertes maternas ocurridas, el 18,2\% fueron desordenes hipertensivos del embarazo, siendo la segunda causa conocida de muerte materna" (12). En el país, la preeclampsia presenta "una incidencia del $7 \%$ de los embarazos y una tasa de mortalidad [para el año 2014] del 42×100.00 nacidos vivos" (13).

Según el boletín epidemiológico de Boyacá, en la semana epidemiológica 52, del año 2017, se contaron 496 casos notificados, lo que representa un incremento del $20 \%(n=81)$ con respecto al año 2016, año en el que se presentaron 415 casos notificados para la misma semana epidemiológica (14).

Por ser la preeclampsia un importante problema de salud prenatal, el objetivo del presente estudio es identificar la prevalencia y los factores asociados a ella, en gestantes de una institución de salud de Boyacá (Mi IPS Boyacá), en los años 2015 a 2017.

\section{MATERIALES Y MÉTODOS}

Se realizó un estudio observacional analítico de corte transversal en gestantes registradas en las bases de datos de una institución de salud (Mi
IPS Boyacá), en el periodo 2015 -2017, en los municipios de Samacá, Villa de Leyva, Moniquirá, Chiquinquirá, Sogamoso, Paipa, Guateque y Garagoa. Este abarcó a 2531 gestantes que asistieron a sus respectivos controles prenatales registrados en las bases de datos de donde se obtuvo la información requerida para el presente estudio; para ese efecto, se contó con la autorización de la directora de la IPS y el comité de Bioética de la Universidad de Boyacá, asimismo, se protegió la privacidad de las participantes evitando publicar los datos personales; de igual forma, se excluyeron las gestantes con datos incompletos en la historia clínica y las que presentaron enfermedades venéreas. Posteriormente, se identificaron las variables del estudio, las cuales fueron:

Edad materna. Para algunos autores, las edades extremas (menor de 20 y mayor de 35 años) constituyen uno de los principales factores de riesgo de hipertensión inducida por el embarazo (THAE), duplicando el riesgo de padecer una preeclampsia. Múltiples conjeturas han tratado de explicar este riesgo incrementado, por ejemplo, se ha planteado que las mujeres mayores de 35 años padecen con mayor frecuencia enfermedades crónicas vasculares, y esto facilita el surgimiento de la PE; Por otra parte, se ha dicho que, en pacientes muy jóvenes, se forman, con mayor frecuencia, placentas anormales, lo cual le da valor a la teoría de la placentación inadecuada como causa de la PE (15). 
Nivel educativo. En esta variable se contempla el nivel de estudios alcanzado por las gestantes en su educación formal, teniendo en cuenta si concluyeron dichos estudios, ya que un bajo grado de educación actúa como factor de riesgo para la PE (16).

Procedencia. Lugar de residencia: urbana (que procede de zona dentro del perímetro urbano de la ciudad), y rural (que procede de pueblos o caseríos aledaños a la ciudad). En un estudio realizado en un hospital de la Amazonia Peruana se encontró que el factor de riesgo asociado a la preeclampsia estuvo asociado a la residencia en zona rural (17).

Factor étnico. Algunos autores informan que la PE aparece con mayor frecuencia en las mujeres afrodescendientes, lo cual ha sido explicado por el hecho de que la hipertensión arterial crónica es más frecuente y severa en estas personas. Además, la obesidad y la diabetes mellitus tipo 2 también son más prevalentes en la población afronorteamericana de los EE.UU. que en la caucásica, y gran parte de los estudios sobre factores de riesgo de PE se han hecho en este país. Es sabido que padecer cualquiera de estas enfermedades crónicas incrementa el riesgo de sufrir una hipertensión inducida por el embarazo (15).

Índice de Masa Corporal (IMC). El riesgo de preeclampsia aumenta de acuerdo con el IMC previo al embarazo. En comparación con las mujeres con un IMC pre embarazo normal $(19,8$ al 26,0), las estimaciones de RR fueron 1,57 (IC del $95 \%: 1,49-1,64)$ y $2,81(95 \% \mathrm{Cl} 2,69-2,94)$, respectivamente, y para las mujeres con sobrepeso (IMC antes del embarazo $=26,1$ a 29,0) y las mujeres obesas (IMC antes del embarazo > $29,0)(18)$.

Riesgo psicosocial. Está demostrada la importancia de la evaluación del riesgo psicosocial durante la primera consulta y a lo largo del control prenatal, para identificar pacientes de alto riesgo de preeclampsia que pueden tener variables psicológicas y sociales de riesgo: embarazos no deseados, ansiedad, humor depresivo, tensión emocional y falta de soporte familiar, entre otros (18).

Presencia de preeclampsia. El diagnóstico debe hacerse a toda embarazada que presente hipertensión (140/90 $\mathrm{mmhg}$ ) después de la semana 20 de gestación, y uno o más de los siguientes hallazgos: proteinuria, insuficiencia renal, enfermedad hepática, alteraciones neurológicas, alteraciones hematológicas o alteraciones fetoplacentarias (17).

Antecedentes reproductivos. No está claro por qué en las primigestas aumenta el riesgo de manera significativa. Una teoría postula que estas pacientes han tenido una exposición limitada a los antígenos paternos, los cuales han mostrado 
tener un papel en la patogénesis de la enfermedad. La nuliparidad se asocia a RR 2.91, 95 \% IC 1.286.6 (18).

Número de controles prenatales. Conjunto de acciones y actividades que se realizan en la mujer embarazada con el objetivo de lograr una buena salud materna. La disminución de la realización de los controles prenatales es un factor de riesgo, dado que no será posible un seguimiento oportuno del embarazo ni detectar sus comorbilidades y complicaciones (17).

Estado del recién nacido. (Prematuridad). Existe un amplio consenso de que uno de los principales trastornos que produce la hipertensión materna es una mayor frecuencia de prematuridad $y$, por lo tanto, una elevada incidencia de neonatos de bajo peso. Existe alrededor de tres veces más riesgo de nacimiento prematuro en las madres con trastornos severos relacionados con la hipertensión arterial (18).

El análisis de los resultados se realizó a partir del software SPSS versión 2.4, usando el análisis de varianza (ANOVA) en busca de probar la hipótesis de que las medias de dos o más poblaciones son iguales, donde evalúa uno o más factores al comparar las medias de la variable de respuesta en los diferentes niveles de los factores. Así mismo, se empleó un análisis multivariado con regresión logística con el fin de calcular el Odds
Ratio (OR) con intervalo de confianza al $95 \%$ y una significancia estadística menor a 0,05.

\section{RESULTADOS}

De las 2531 gestantes que se registraron en la base de datos, la prevalencia de preeclampsia fue de 3,5\%. En la Tabla 1 se muestran las variables del estudio, encontrando la edad promedio en tres rangos así: mujeres $<20$ años con preeclampsia pertenecen a una media de 16,13 y sin preeclampsia a una media de 17,78 ; en el rango de 25 a 35 años, las gestantes que presentan preeclampsia se encuentran en el promedio de 26,62 y sin preeclampsia en un promedio de 27,48; gestantes mayores de 35 años con preeclampsia pertenecen a una media de 40,62 y sin ella una media de 38,26 . Para el IMC, se evidenció un promedio de $20,69 \mathrm{~kg} / \mathrm{m}^{2}$ en un rango normal en las gestantes con diagnóstico de preeclampsia, y un IMC de $20,20 \mathrm{~kg} / \mathrm{m}^{2}$ en un rango normal en las gestantes que no tenían preeclampsia. El número de controles prenatales está en un promedio de 7,52 en las gestantes que padecen de preeclampsia, y una media de 8,67 en las gestantes que no. 
Tabla 1. Características de las gestantes

\begin{tabular}{ccc}
\hline Factor de Riesgo & $\begin{array}{c}\text { Preeclampsia } \\
\text { MEDIA (DS) }\end{array}$ & $\begin{array}{c}\text { NO preeclampsia } \\
\text { MEDIA (DS) }\end{array}$ \\
\hline EDAD & \\
\hline$<20$ años & $16,13(0,806)$ & $17,76(1,335)$ \\
\hline $20-35$ años & $26,62(4,681)$ & $27,48(4,313)$ \\
\hline$>35$ años & $40,62(3,530)$ & $38,26(2,032)$ \\
\hline IMC & $20,69(4,032)$ & $20,20(3,666)$ \\
\hline Número de controles & $7,52(2,825)$ & $8,67(1,584)$ \\
\hline
\end{tabular}

En la Tabla 2 se muestra que todos los factores de riesgo resultaron estadísticamente significativos para presentar preeclampsia. Entre los factores que se destacaron están: presentar un riesgo biopsicosocial alto (63\%) y; estado del recién nacido prematuro y con bajo peso al nacer (43\%).

Tabla 2. Prevalencia de factores de riesgo significativos en las gestantes

\begin{tabular}{cccc}
\hline Factor de riesgo & $\begin{array}{c}\text { Preeclampsia } \\
\mathbf{n}=\mathbf{9 0}(\%)\end{array}$ & $\begin{array}{c}\text { NO preeclampsia } \\
\mathbf{n = 2 4 4 1}(\%)\end{array}$ & P \\
\hline PROCEDENCIA & & & \\
\hline 1. URBANA & $81(90 \%)$ & $2324(95,2)$ & $0,0206^{*}{ }^{\prime}$ \\
\hline 2. RURAL & $9(10 \%)$ & $117(4,8 \%)$ & \\
\hline RIESGO BIOPSICOSOCIAL & & \\
\hline 1. ALTO & $57(63,3 \%)$ & $1092(44,7 \%)$ & $0,000^{*}$ ŕ \\
\hline 2. BAJO & $33(36,7 \%)$ & $1349(55,3 \%)$ & \\
\hline
\end{tabular}

\begin{tabular}{|c|c|c|c|}
\hline \multicolumn{4}{|c|}{ ESTADO DEL RECIÉN NACIDO } \\
\hline 1. A TÉRMINO & $39(43,3 \%)$ & $1974(80,9 \%)$ & $0,000^{*}{ }_{\Gamma}^{\prime}$ \\
\hline $\begin{array}{l}\text { 2. MACROSO- } \\
\text { MÍA }\end{array}$ & & $9(0,4 \%)$ & \\
\hline 3. PREMATURO & & $26(1,1 \%)$ & \\
\hline $\begin{array}{l}\text { 4. PREMATU- } \\
\text { RO, BAJO PESO }\end{array}$ & & $26(1,1 \%)$ & \\
\hline $\begin{array}{l}\text { 5. PREMATU- } \\
\text { RO, BAJO PESO } \\
\text { AL NACER }\end{array}$ & $39(43,3 \%)$ & $7(0,3 \%)$ & \\
\hline $\begin{array}{l}\text { 6. PRETÉRMI- } \\
\text { NO }\end{array}$ & $12(13,3 \%)$ & $339(16,3 \%)$ & \\
\hline
\end{tabular}

${ }^{*}$ Anova. $\mathrm{P}<0.05$

Entre las gestantes que presentan el nivel educativo de tecnología, se encuentra que el 36,7 \% de ellas presentaban preeclampsia. El $64 \%$ de las gestantes se realizaron 7 controles prenatales durante toda su gestación.

En la Tabla 3 se muestra el análisis del modelo de regresión logística entre los factores de riesgo y la presencia de preeclampsia. Las gestantes con más de 35 años tuvieron 5,2 veces más probabilidades de tener preeclampsia en comparación con las que tenían menos de 35 años (OR: 5,2; IC 95 $\%: 3,258-8,335)$. Las gestantes que tienen riesgo psicosocial alto tuvieron 2,1 veces más probabilidad de presentar preeclampsia en comparación con las que tienen un riesgo psicosocial bajo (OR: 2,134; IC 95\%: 1,380- 3,300). 
Tabla 3. Asociación entre factores de riesgo y preeclampsia

\begin{tabular}{cccc}
\hline Factor de riesgo & OR & IC & P \\
\hline EDAD & - & - & $0,000^{*}$ ŕ \\
\hline$<20$ AÑOS & 1,537 & $0,836-2,827$ & $0,229^{*}$ \\
\hline 20-35 AÑOS & 5,211 & $3,258-8,335$ & $0,000^{*}$ ŕ \\
\hline >35 AÑOS & & & \\
\hline RIESGO PSICOSOCIAL & & 0,001 ŕ \\
\hline 1. ALTO & 2,134 & $1,380-3,300$ & - \\
\hline
\end{tabular}

*Anova, $\mathrm{P}<0.05$

\section{DISCUSIÓN}

De acuerdo con los resultados, es posible observar que a medida que la edad materna es mayor, el riesgo de padecer preeclampsia aumenta, siendo importante valorar la edad materna y su estrecha relación con el riesgo de desarrollar la patología. Autores como Suárez González, afirman que el mayor índice de preeclampsia apareció en el grupo mayor de 35 años (19). Según Benites Cóndor Yamalí, este resultado puede deberse a que las mujeres mayores de 35 años tienen mayor frecuencia de enfermedades crónicas, y además se puede explicar por una posible isquemia placentaria secundaria al aumento de lesiones escleróticas del miometrio (4); por otra parte, Valdes
Yong, afirma que, la preeclampsia puede afectar a mujeres mayores de 35 años debido a los daños crónicos del sistema vascular que sufre desgastes, con la consecuente esclerosis de los vasos; lo anterior, afecta el aporte sanguíneo adecuado durante el embarazo y causa insuficiencia circulatoria, produciendo una isquemia útero-placentaria (20). De igual manera, el estudio de Rojas, et al. reporta que superar los 35 años es un factor de riesgo para las gestantes, pues dicha asociación representa un riesgo incrementado para preeclampsia, lo cual puede contribuir a la aparición de otras complicaciones maternas (21).

Así mismo, Martel L., afirma que tener más de 35 años representa uno de los factores de riesgo más importantes para desarrollar la patología (22). Por su parte, Başer en el que se evidencia que la edad materna avanzada aumenta el riesgo de complicaciones perinatales y de resultados adversos del embarazo, en comparación con mujeres más jóvenes. De esta forma, se corrobora que los riesgos en edad avanzada comienzan a aumentar después de la edad de 35 años (23).

Respecto a la presencia de factores psicosociales y mayor riesgo de presentar preeclampsia, la situación se contrasta con los resultados obtenidos en la investigación que realizó K. Elia, en la cual se valora que la preeclampsia sigue siendo un problema relevante de salud pública en el que influyen factores psicosociales, los cuales deben ser 
considerados, evaluados y atendidos para disminuir sus efectos sobre la salud materna y perinatal (24); esta posición coincide con la Vega Morales, quien refiere en su estudio, que la preeclampsia es una enfermedad multifactorial del embarazo, y los factores psicosociales son un componente fundamental para considerar en su etiología (25).

Es importante resaltar que en el presente estudio tuvo como limitación la calidad de la información registrada en las historias clínicas de las pacientes, dado que los antecedentes de enfermedad y los antecedentes reproductivos en los tres rangos de edad no se encontraron diligenciados; lo anterior, impidió registrar enfermedad y antecedente reproductivo.

\section{CONCLUSIÓN}

Es la edad extrema superior a los 35 años el factor de riesgo predominante para desarrollar preeclampsia, asociado posiblemente al padecimiento de enfermedades crónicas vasculares durante la misma etapa de vida, aumentando su presentación y progreso con el embarazo.

Se recomienda brindar educación a la población sobre los riesgos de la gestación en edades extremas de la edad fértil, con estrategias como la planificación familiar y las consultas preconcepcionales, establecidas en las Rutas de atención en Salud (RIAS), con el fin de que toda mujer en edad fértil obtenga algún método anticonceptivo hasta que por decisión propia desee un embarazo.

Las mujeres en estado de gestación se convierten en una población con mayor vulnerabilidad frente a sus condiciones laborales, económicas y el desempeño de sus actividades cotidianas, más aún si presentan patologías asociadas a su condición. De acuerdo con los resultados del presente estudio, es necesario promover estrategias de intervención en la gestante, con el fin de fomentar e identificar sus derechos y garantizar su cumplimiento, así también, aclarar sus dudas y fomentar su participación en busca de apoyar sus decisiones y mejorar sus condiciones psicosociales.

Conocer el estado actual de cada usuaria ante la decisión del embarazo favorece la atención personalizada, la prevención en riesgos y la condición de la mujer para afrontar su gestación, mejorando la calidad de vida y la salud de las gestantes y sus familias.

\section{AGRADECIMIENTOS}

Una investigación es el resultado de ideas, proyectos y esfuerzos; por eso, deseamos expresar nuestros agradecimientos a la Universidad de Boyacá y a Mi IPS Boyacá por el apoyo que brindaron a este trabajo y por la orientación recibida. 


\section{FINANCIACIÓN}

Recursos propios de los autores y Universidad de Boyacá

\section{CONFLICTO DE INTERESES}

No existe algún conflicto de intereses por los posibles resultados.

\section{REFERENCIAS}

1. Lisonkova S, Sabr Y, Mayer C, Young C, Skoll A, Joseph KS. Maternal Morbidity Associated with Early-Onset and Late-Onset Preeclampsia. Obstetrics \& Gynecology. 2014;124(4):771-781. http://dx.doi: 10.1097/ AOG.0000000000000472

2. Nápoles Méndez D. New interpretations in the classification and diagnosis of pre-eclampsia. Medisan. 2016;20(4):517. Recuperado a partir de: http://www.medigraphic.com/pdfs/ medisan/mds-2016/mds164m.pdf

3. Guzmán-Juárez W, Ávila-Esparza M, Contreras-Solís $\mathrm{RE}$, Levario-Carrillo $\mathrm{M}$. Factores asociados con hipertensión gestacional y Preeclampsia. Ginecol Obstet Mex 2012;80(7):461466. Recuperado a partir de: http://www. medigraphic.com/pdfs/ginobsmex/gom-2012/ gom127d.pdf
4. Benites $Y$, Razan S. Factors associated with the development of pre-eclampsia in a hospital of Piura, Peru. Cimel. 2011;16(2):77-82. Recuperado a partir de: https://es.scribd.com/document/363775039/Factores-Asociados-Al-Desarrollo-de-Preeclampsia-en-Un-Hospital-de-Piura

5. Keskinkılıç B, Engin-Üstün Y, Sanisoğlu S, Şahin Uygur D, Keskin HL, Karaahmetoğlu S, Özcan $A$, et al. Maternal mortality due to hypertensive disorders in pregnancy, childbirth, and the puerperium between 2012 and 2015 in Turkey: A nation-based study. Journal of the Turkish German Gynecological Association. 2017;18(1):20-25. http://dx.doi: 10.4274/ jtgga.2016.0244

6. Programa de las Naciones Unidas para el Desarrollo. Objetivos del desarrollo sostenible. One United Nations Plaza 2010. Recuperado a partir de: http://www.co.undp.org/content/ colombia/es/home/sustainable-development-goals.html

6. Vargas VM, Acosta G, Moreno MA. La Preeclampsia un problema de salud pública mundial. Rev. chil. obstet. ginecol. 2012;77(6):471-476. http://dx.doi: 10.4067/ s0717-75262012000600013. 
7. López Mora J. Preeclampsia-eclampsia. Revista Latinoamericana de Hipertensión 2007;2(4):117-127. Sociedad Latinoamericana de Hipertensión Caracas, Organismo Internacional. ISSN: 1856-4550. Recuperado a partir de: http://www.redalyc.org/articulo. oa? id $=170216979004$

8. OMS. Mortalidad materna. 2016 Disponible en: http://www.who.int/mediacentre/factsheets/fs348/es/ (último acceso 26 de septiembre de 2017)

9. Herrera JA, Herrera-Miranda R, Herrera-Escobar JP, Nieto-Díaz A. Reducción de la mortalidad materna por Preeclampsia en Colombia - un análisis de series de tiempo interrumpido. Colombia Médica 2014; 45(1): 25-31. http://www.scielo.org.co/pdf/cm/v45n1/es_ v45n1a04.pdf

10. Agudelo MD, Agudelo LM, Castaño JJ, Giraldo JF. Prevalencia de los trastornos hipertensivos del embarazo en mujeres embarazadas controladas en Assbasalud E.S.E, Manizales (Colombia), 2006 a 2008. Universidad de Manizales. 10(2): Dic. 2010. Recuperado a partir de: DOI: https://doi.org/10.30554/ archmed.10.2.1293.2010

11. Lopera Rodríguez JA, Rocha Olivera E. Preeclampsia: su asociación con infecciones perio- dontales y urinarias según trimestre del embarazo. CES Med. 2016; 30(1): 14-25. https://doi. org/10.21615/cesmedicina.30.1.2

12. Beltrán Chaparro LV, Benavides $P$, López Rios JA, Onatra Herrera W. Estados hipertensivos en el embarazo. Rev. U.D.C.A Act. \& Div. Cient 2014; 17(2): 311-323 http://www.scielo.org. co/pdf/rudca/v17n2/v17n2a02.pdf

13. Dirección Técnica de Salud Pública Vigilancia en Salud Pública. Boletín Epidemiológico de Boyacá. 2017; (52). http://www.boyaca.gov. co/SecSalud/component/easyfolderlistingpro

14. Cruz Hernández J, Hernández García P, Yanes Quesada M, Isla Valdés A. Factores de riesgo de Preeclampsia: enfoque inmunoendocrino. Parte I. Revista Cubana de Medicina General Integral. 2007; 23(4). ISSN 1561-3038. Recuperado a partir de: http:// scielo.sld.cu/scielo.php?script =sci_arttext\&pi$d=$ S0864-21252007000400012\&lng =es\&tIn$g=e s$

15. Gozar Casas MA. Factores de riesgo asociados a Preeclampsia leve en mujeres primigestas en el hospital de vitarte en el año 2015. Universidad Ricardo Palma. 2017 http://cybertesis. urp.edu.pe/bitstream/urp/935/1/Gozar\%20 Casas\%20Miguel\%20\%C3\%81ngel_2017.pdf 
16. Alcaldía Mayor de Bogotá. Guía de trastornos hipertensivos en el embarazo. Bogotá Humana. 2013. http://www.saludcapital. gov.co/DDS/Publicaciones/Guia\%20Maternidad-Trastornos_baja.pdf

17. Torres S. Factores de riesgo para Preeclampsia en un hospital de la amazonia peruana. CASUS. 2016. http://casus.ucss.edu.pe/index. php/casus/article/view/11/6

18. Suárez González JA, Cabrera Delgado MR, Gutiérrez Machado M, Corrales Gutiérrez A, Cairo González V, Rodríguez Royelo L. Resultados de la atención a pacientes con riesgo de Preeclampsia-eclampsia. Rev Cubana Obstet Ginecol. 2012;38(3): 305-312. http:// scielo.sld.cu/scielo.php?script=sci_arttext\&pi$d=$ S0138-600X2012000300003\&Ing=es

19. Váldes Yong Ml, Hérnandez Núñez J. Factores de riesgo para preeclampsia. Revista Cubana de Medicina Militar. 2014; 43(3): 307-316. http://scielo.sld.cu/pdf/mil/v43n3/mil05314.pdf

20. Rojas FA, Flores ER, Valencia DJ, Correa LE. Preeclampsia y sus principales factores de riesgo. Rev. Fac. Med. Hum. 2017; 17(2): 90-99. http://dx.doi 10.25176/RFMH.v17.n2.839.

21. Martel LM, Ovejero SC, Gorosito IC. Preeclampsia y factores de riesgo en embarazadas en el Instituto de Maternidad y Ginecologia Nuestra Señora de las Mercedes en Tucumán. Universidad Nacional de Tucumán. Intramed Journal. 2010; 1(3). http://journal.intramed. net/index.php/Intramed_Journal/article/viewFile/179/43

22. Başer $E$. The impact of parity on perinatal outcomes in pregnancies complicated by maternal age. J Turk Ger Gynecol Assoc. 2013; 14(4): 205-209. http://dx.doi:10.5152/ jtgga.2013.62347

23. Ku E. Factores psicosociales asociados a Preeclampsia en mujeres hospitalizadas en el Instituto Nacional Materno Perinatal, agosto a octubre de 2012. Revista Peruana de Epidemiología. 2014;18(1):1-7. Sociedad Peruana de Epidemiología Lima, Perú. http://www.redalyc. org/pdf/2031/203131355009.pdf

24. Vega-Morales EG, Torres-Lagunas MA, Patiño-Vera V, Ventura-Ramos C, Vinalay-Carrillo I. Vida cotidiana y Preeclampsia: Experiencias de mujeres del Estado de México. Enferm. univ. 2016; 13(1): 12-24. http://dx.doi. org/10.1016/j.reu.2015.11.006

Esta obra está bajo una licencia de Creative Commons Reconocimiento-NoComercial 4.0 Internacional 